\title{
Transarterial Therapies for Hepatocellular Carcinoma: a Comprehensive Review with Current Updates and Future Directions
}

\author{
Yogesh Kumar $^{1 *}$, Pranav Sharma ${ }^{2}$, Neeraj Bhatt ${ }^{1}$, Kusum Hooda $^{1}$
}

\begin{abstract}
Hepatocellular cancer is a very common cause of cancer related deaths worldwide. Only 30-40\% of patients present with early-stage disease open to curative treatments, such as resection or transplantation, while others can only undergo local therapies or palliative care. Various trans-arterial approaches have been used for treatment of hepatocellular carcinoma in patients who need a down-staging to liver transplantation, and who are not candidates for transplantation or radiofrequency ablation. Transarterial chemoembolization (TACE), transarterial embolization (TAE), drug-eluting beads, and radioembolization have been used for locoregional control, and have been shown to prolong the overall survival when compared with supportive care. In this review, we discuss patient selection, pre- and post-procedure imaging, techniques, safety, and clinical outcomes related to these therapies. Newer advances with future directions in various fields related to trans-arterial therapies are also discussed.
\end{abstract}

Keywords: Embolization - chemoembolization - hepatocellular carcinoma - chemotherapy - transarterial treatment

Asian Pac J Cancer Prev, 17 (2), 473-478

\section{Introduction}

Hepatocellular Carcinoma (HCC) is the most common primary malignant cancer affecting the liver. It is the fifth and seventh type of cancer that affects men and women respectively. Additionally, $\mathrm{HCC}$ is categorized as the second common cause of cancer deaths among men and sixth common cause of cancer death among women (Jemal et al., 2011). HCC most commonly occurs in patients with underlying chronic liver disease including cirrhosis. While alcohol is the most common cause of cirrhosis in developed worlds, hepatitis B and C infections are more common cause in the developing countries including some parts of Asia and Africa (Can et al., 2014; Khan et al., 2015).

As the treatment of HCC depends on the stages of disease, many prognostic systems have been developed to stratify the patients. The most commonly used systems include Child-Pugh, Okuda, Barcelona Clinic Liver Cancer, Japan Integrated Scoring, and Tumor-NodeMetastasis (Llovet et al., 1999). The Child-Pugh scoring system is often used to determine hepatic reserve, but Barcelona Clinic Liver Cancer system is more often used to determine the treatment as it includes the extent of disease and underlying liver function (Zhang et al., 2014). Patients with early-stage HCCs (Barcelona Clinic Liver Cancer class A) are further categorized in to two categories. Patients with preserved liver function (either
Child-Pugh class A or B) can undergo treatment with lesion resection or percutaneous methods of treatment such as radiofrequency ablation (RFA) particularly in elderly patients or those with comorbidities precluding surgery or transplant (Yang et al., 2014). In patients with early-stage HCCs but poor liver function, and single tumor less than $5 \mathrm{~cm}$ in largest diameter or up to three tumors less than $3 \mathrm{~cm}$ each without evidence of vascular or lymphatic invasion, liver transplant have been shown to have excellent outcomes (Xie et al., 2015). Intraarterial therapy has been used for patients with intermediate- stage HCC (Barcelona Clinic Liver Cancer class B. The only systemic treatment that has shown a benefit for patients with late-stage HCC (Barcelona Clinic Liver Cancer class C) is sorafenib, a multikinase inhibitor (Llovet et al., 2008). However, in select cases of Barcelona Clinic Liver Cancer class $\mathrm{C}$ disease, transarterial therapies have also been used. Various transarterial therapies include TACE, TAE, drug-eluting beads, and radioembolization, which will be discussed in detail including newer advances with future directions in various fields.

\section{Technical Considerations in General}

Transcatheter intraarterial therapies are based on the fact that most of hepatic neoplasms preferentially derive their blood supply from an arterial source (mainly hepatic artery), while rest of the liver gets its blood supply mainly 


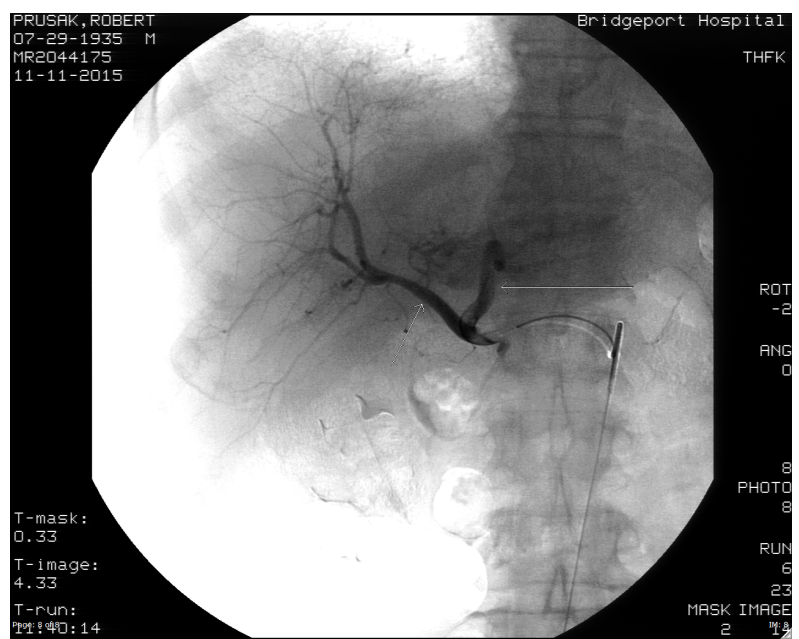

Figure 1. Pre-procedural angiographic image demonstrated HCC being supplied mainly by left hepatic artery ( larger arrow), and right hepatic artery (smaller arrow) supplying the normal liver

A

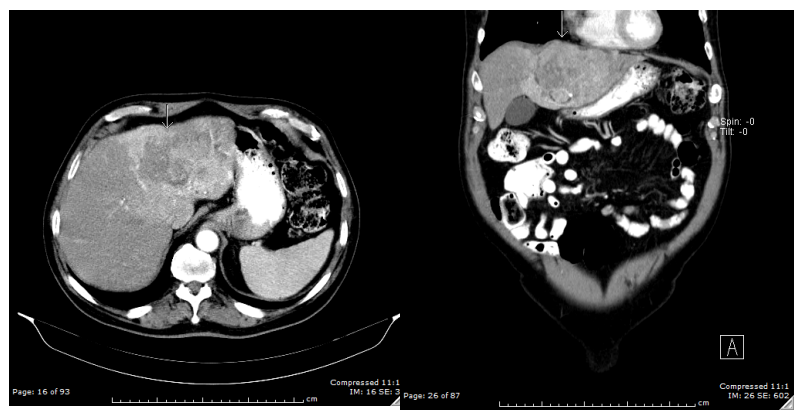

Figure 2. Axial (A) and coronal (B) contrast enhanced CT in the arterial phase demonstrates hyper enhancing hepatocellular carcinoma (marked by arrows)

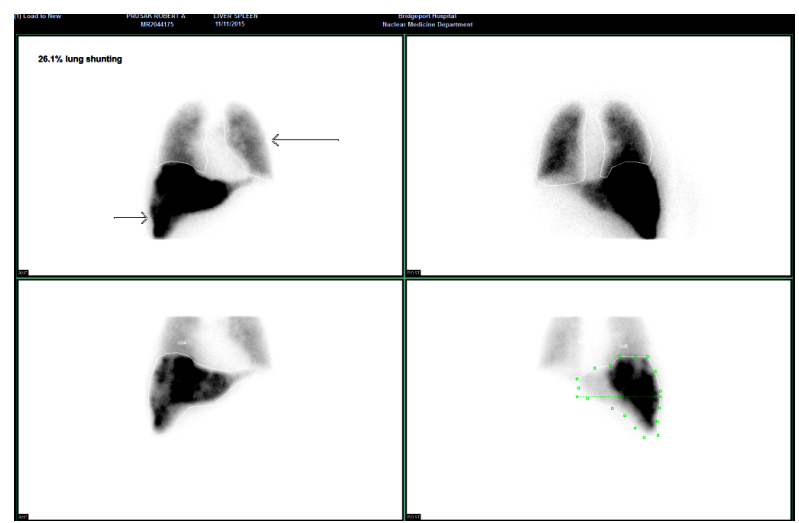

Figure 3. Nuclear medicine study with $99 \mathrm{~m}$ Tcmacroaggregated albumin showed a $26 \%$ liver (smaller arrow) to lung (larger arrow) shunt leading to reduction in dose of $\mathbf{Y 9 0}$ to decrease radiation dose to the lungs

from the portal vein. This allows for selective therapy delivery to hepatic tumors and protects against ischemic necrosis of the rest of the liver. Previously thought to be a contraindication for hepatic arterial embolization procedures, recent data has revealed a highly selective

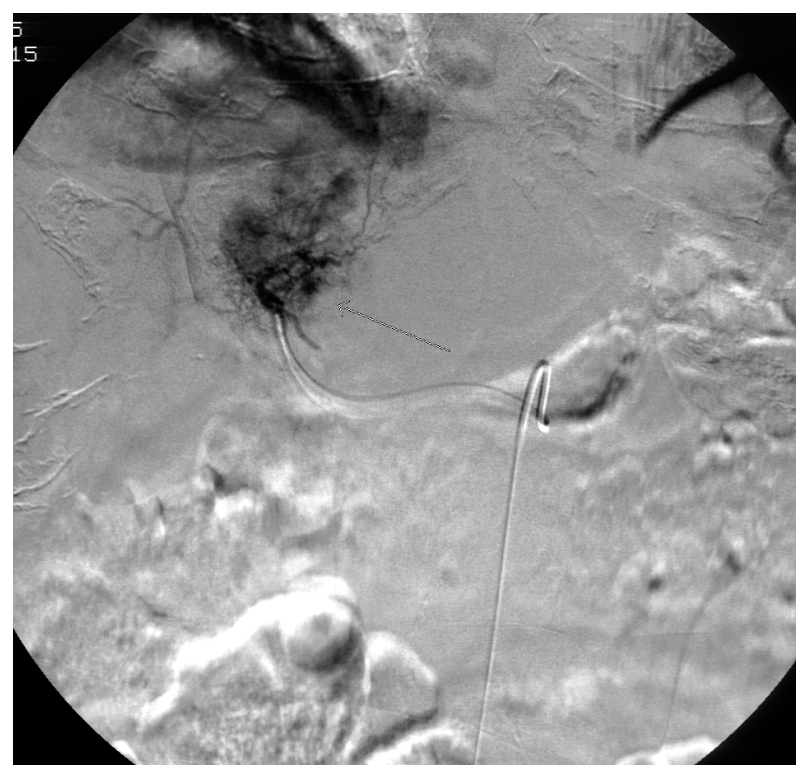

Figure 4.Angiographic image during radio embolization in the same patient showed delivery of the Y90 to the HCC through the left hepatic artery (arrow)

approach may be safe in patients with portal vein thrombosis (Luo et al., 2011). Radioembolization, which is a much less embolic procedure, has been proven safe and effective in patients with portal vein thrombosis (Kulik et al., 2008; Iñarrairaegui et al., 2010).

The techniques for trans arterial therapies are widely variable, and both operator and institution dependent. The common femoral artery is usually chosen for access. For appropriate targeted therapy delivery and the prevention of complications, knowledge of variant hepatic anatomy is very important for all transcatheter therapies. Specifically, awareness of extrahepatic vessels arising from the hepatic arteries, and the prospective identification of these nontarget vessels can minimize the risk of adverse events related to the extrahepatic deposition of the therapeutic agent from nontarget embolization (Covey et al., 2002; Song et al., 2006). Therefore, diagnostic angiography is routinely performed in the celiac artery and superior mesenteric artery to define the normal and variant arterial anatomy ( Figure 1).

Radioembolization is potentially thought to be one of the more technically challenging of the embolization procedures because of the risk of nontarget embolization. However, it is now being recognized that when delivered to nontarget vascular territories, all loaded embolic therapies can result in severe adverse events. With more experience with drug-eluting beads, more and more adverse events are now being identified (Liapi et al., 2011). Thus, detailed angiography and identification of collaterals is of paramount importance prior to all transcatheter intraarterial therapies.

\section{Individual Approaches}

As previously mentioned, transarterial therapies for hepatocellular carcinoma mainly consists of TACE, TAE, drug-eluting beads, and radioembolization, which will be discussed in detail (Ma et al., 2013). 
Transarterial Therapies for Hepatocellular Carcinoma: a Comprehensive Review with Current Updates and Future Directions

\section{Transarterial Chemoembolization}

TACE is usually considered in patients who are not eligible for curative resection or ablation, and have Barcelona Clinic Liver Cancer class B or intermediate stage (Okuda stage I-II) disease (Llovet et al., 1999). Although studies have shown improved survival in patients with Child-Pugh class A disease and albumin level greater than $3.4 \mathrm{~g} / \mathrm{dL}$ when treated with TACE in comparison with those with Child-Pugh class B or C disease, there are no definitive exclusion criteria based on laboratory data currently (Brown et al., 2004). Although considered a contraindication for TACE, studies have shown that the patients with portal vein thrombosis can be safely treated and there has been no significant difference in mortality between patients with patent portal veins and segmental portal vein thrombosis treated with TACE $(\mathrm{Xu}$ et al., 2014).

The absolute contraindications to TACE include decompensated cirrhosis, severely reduced portal vein flow, extensive involvement of both lobes with tumor, and renal insufficiency with creatinine clearance less than 30 $\mathrm{mL} / \mathrm{min}$. Comorbidities with compromised organ function, such as heart disease and lung disease, Tumors larger than $10 \mathrm{~cm}$, and untreated varices with risk of bleeding have been described as relative contraindications.

In clinical practice, single, dual and triple-agent protocols have been used for TACE. Doxorubicin is the most commonly used agent followed by cisplatin, while mitomycin $\mathrm{C}$ is added for triple agent therapy (Marelli et al., 2007). Many prospective randomized control trials have shown no clear advantage of one over another agent (Chang et al., 1994), and therefore, the choice of chemotherapeutic agent depends upon individual preferences and remains variable. Single-agent doxorubicin is being commonly used worldwide, whereas triple agent therapy using the combination of mitomycin C, doxorubicin, and cisplatin is being used in the United States (Kong et al., 2012).

TACE consists of infusion of potent chemotherapeutic agents into the hepatic arteries supplying the tumor in an emulsion created using ethiodized oil, followed by embolization with particles such as gel- foam, polyvinyl alcohol, or acrylic co- polymer gelatin particles (Coldwell et al., 1994). An emulsion created using ethiodized oil and chosen chemotherapeutic agent is taken up and retained by HCC but cleared out by Kupffer cells in normal liver. This mechanism is thought to increase the concentration of the chemotherapeutic agent with in the tumor (Kan et al., 1994). The purpose of embolization is to prevent washout of the drug from the tumor and to induce ischemic necrosis. Furthermore, the necrosis might allow a greater absorption of chemotherapeutic agents by tumor cells due to failure of transmembrane pumps in tumor cells (Kruskal et al., 1993). Thus, TACE exposes the tumors to high concentrations of local chemotherapeutic agents, while keeping the systemic drug levels minimal.

Routine pre- procedure imaging consists of a multiphase CT or MRI of the liver (Figure 2). The main purposes of pre- procedure imaging is look for variant arterial anatomy, enlarged or tortuous intercostal, inferior phrenic, and internal mammary arteries, portal vein tumor thrombus, and the presence of extrahepatic disease (Covey et al., 2002). It is also important to note the presence of bilioenteric anastomoses, stents, or the presence of gas within the biliary tree, possibly resulting from previous sphincterotomy in order to decrease the incidence of post- procedure hepatic abscess, because patients without an intact sphincter of Oddi have biliary tree colonized by bacteria from gastrointestinal tract (Kim et al., 2001).

Post procedure imaging for TACE can be done with either multiphasic CT or MRI. However, due to increased density of the ethiodized oil in the tumor, evaluation of recurrent or residual tumor enhancement in post procedure CT can be a challenge. Therefore, for patients treated with TACE, most practitioners prefer MRI to CT for follow-up after TACE (Kloeckner et al., 2010).

The post embolization syndrome is the most common post procedure event after TACE, which occurs in 60-80\% of patients, and includes fever, pain, nausea, transaminitis, and vomiting (Shi-Ying et al., 2013). Other complications are very uncommon and include biloma, abscess formation, cholecystitis, arterial dissection, hepatic failure, and gastrointestinal bleed (Marelli et al., 2007).

\section{Transarterial Embolization}

TAE has similar patient selection criteria to those for TACE and includes Barcelona Clinic Liver Cancer class B disease or intermediate stage (Okuda stage I-II) disease. Similar to TACE, there are no definitive laboratory exclusion criteria and, patients with portal vein thrombosis have been shown similar survival when compared with similar patients treated with TACE (Deodhar et al., 2010). However, patients with preexisting cardiovascular problems have shown increased morbidity.

Similar to TACE, decompensated cirrhosis, severely reduced portal vein flow, extensive involvement of both lobes with tumor, and renal insufficiency with creatinine clearance less than $30 \mathrm{~mL} / \mathrm{min}$ have been described as absolute contraindications to TAE, and relative contraindications include tumors larger than $10 \mathrm{~cm}$, comorbidities with compromised organ function, such as heart disease and lung disease, and untreated varices with risk of bleeding.

The technique for TAE varies among individuals and institutions. The main purpose of TAE is complete stasis of the vessels supplying the tumors leading to ischemia-induced death of the tumor cells. This can be performed using embolic agents varying in size and physical capabilities. These include small-calibrated microspheres, absorbable gelatin sponge, and polyvinyl alcohol. However, small particles are usually favored over larger particles as with larger particles proximal vessel blockade may induce inadequate hypoxia, rather than severe hypoxia or anoxia achieved with terminal vessel blockade, which is achieved with smaller particles. Studies in the past have shown that inadequate hypoxia can lead to increased cell survival through the expression of hypoxia-inducible factor.

As with TACE, routine pre- procedure imaging with TAE also consists of a multiphase CT or MRI of the liver with same purposes of looking for variant arterial anatomy and supply, portal vein tumor thrombus, the presence of 
extrahepatic disease; and the presence of bilioenteric anastomoses, stents, or the presence of gas within the biliary tree tract (Kim et al., 2001). Since there are no lasting hyperdense changes obscuring tumor enhancement with TAE, multiphase CT alone is usually adequate for detecting enhancement after TAE. However, it is important to remember that contrast agent can be retained in the tumor up to several days after small-particle TAE.

With the exception that chemotherapy-related side effects are not seen with TAE, complications for TAE are similar to those for TACE. In addition, presence of hepatic arterial-systemic venous shunts in patients undergoing small-particle TAE using 40-120- $\mu$ m tris-acryl gelatin microspheres have resulted in fatal pulmonary vessel blockade. Pancreatitis, pulmonary edema, and cardiac arrhythmias also have been reported.

\section{Drug eluting microspheres}

The indications and contraindications for drug eluting microspheres are the same as conventional TACE and TAE. The basic concept behind chemoembolization with drug-eluting beads is to load polyvinyl alcohol-based microspheres with various types of chemotherapeutic agents followed by intra-arterial delivery similar to conventional chemoembolization. This mechanism enhances the delivery of potent anticancer agents to the tumor site. Sustained and controlled release of the anticancer agents from the drug eluting microspheres allows for fixed dosing to the tumor. Moreover, when compared with conventional chemoembolization, there have been significant reductions of peak plasma concentrations with drug-eluting beads (Bruix et al., 2011). This diminishes the systemic bioavailability of the agent while a greater amount of the anticancer agent is being delivered to the tumor resulting in a more pronounced tumor response (Hong et al., 2006). Doxorubicin is the drug being used most commonly for the treatment of HCC. Currently, two most common drugeluting beads being used are LC Beads and Quadraspheres. Both of these result in high tumoral concentrations of the loaded chemotherapeutic agents and have similar rates of tumor necrosis with lower plasma concentrations and less effect on normal liver. For these reasons, lower hepatic and chemotherapy-related toxicity has been reported with drug-eluting microspheres when compared with conventional TACE (Lammer et al., 2010). Similar to TAE, multiphase CT alone is usually adequate for detecting enhancement after treatment with drug eluding microspheres.

\section{Radioembolization}

A multidisciplinary team approach is usually necessary when selecting patients for radioembolization. A consensus panel consisting of experts from medical oncology, surgical oncology, nuclear medicine, interventional radiology, and radiation oncology have outlined patient selection criteria for radioembolization (Kennedy et al., 2007). Patients who are not resection candidates and who have a life expectancy longer than 3 months should be considered. Contraindications include pulmonary shunts that may result in a radiation dose of 30 Gy or greater to the lungs or extrahepatic flow to the gastrointestinal tract that cannot be corrected via catheter embolization, total serum bilirubin greater than $2 \mathrm{mg} / \mathrm{dL}$, severely reduced portal vein flow, and poor hepatic reserve (Kennedy et al., 2007).

Two different radioembolization agents, SIR-Spheres and Thera- sphere are most commonly used. Therasphere is used for the treatment of unresectable HCC with and without portal vein occlusion. In addition to the contraindications listed above, caution has been advised for use of Thera-sphere in patients with infiltrative tumor, tumor volume comprising more than $70 \%$ of the target liver volume, tumor volume $50 \%$ with an albumin level less than $3 \mathrm{~g} / \mathrm{dL}$, or with innumerable tumor nodules. A pulmonary shunt over $20 \%$ and treatment with capecitabine anytime after SIR-Spheres or in the 2 months before SIR-Spheres treatment have been mentioned as contraindications to radioembolization with SIR-Spheres (Figure 3).

The radioembolization involves the delivery of radioactive microspheres to hepatic tumor, and thus is different than that for TAE or TACE ( figure 4). The radioactive microspheres emit $\beta$-radiation to the surrounding tissues via the decay of $90 \mathrm{Y}$, which is a pure $\beta$ emitter. The treatment relies on the delivery of radioactive microspheres preferentially to the tumors related to the hepatic arterial flow of tumors. Because of its predominant blood supply arising from the portal vein, the normal liver receives a much lower dose.

The procedure is divided into two portions performed on separate days, a hepatic arterial mapping day and a treatment day. Extrahepatic flow and lung shunt fraction are evaluated with hepatic arteriography with embolization of extrahepatic of 99mTc-macroaggregated albumin. If no significant extra hepatic flow is detected, the patient returns for radioembolization treatment. Since the microspheres used for radioembolization cannot be mixed with contrast material, targeted delivery requires a meticulous preparation and technique.

Contrast enhanced CT or MRI can be used to perform follow-up imaging after radioembolization. Other than the usual imaging findings seen with TACE and TAE, including necrosis, ascites, pleural effusion, and perihepatic fluid, important additional findings with $90 \mathrm{Y}$ therapy include hepatic fibrosis and ring enhancement (Atassi et al., 2008). Ring enhancement can sometimes be seen which represents granulation tissue and fibrosis. Diffusion-weighted MR imaging has shown some promise in early detection of response in HCC, and could be considered.

Since radioembolization uses radiation, its complications are distinctly different from those seen with TACE and TAE. These mainly include abscess, portal hypertension, radiation pneumonitis, gastrointestinal ulcers, and radiation-induced liver disease. A postradioembolization syndrome including fatigue, weakness, nausea, anorexia has been described that can last up to 3 weeks after the procedure and is usually selflimited. 
Transarterial Therapies for Hepatocellular Carcinoma: a Comprehensive Review with Current Updates and Future Directions

\section{Outcomes and Comparison of Transcatheter Therapies}

There are only few studies in the literature, which have effectively compared transcatheter therapies with each other and supportive care. Llovet et al (2002) and Lo et al (2002) in two different randomized controlled trials showed a statistically significant survival benefit in patients under- going TACE versus supportive care.

On comparing three randomized controlled trials, Marelli et al concluded that transarterial chemoembolization failed to demonstrate a survival benefit compared with transarterial embolization alone. However, a recent prospective randomized control study comparing the chemoembolization with doxorubicin-eluting beads and arterial embolization without doxorubicin for HCC showed an additional benefit from the addition of doxorubicin.

Kooby et al found no significant difference in effectiveness and toxicity of either chemoembolization or radioembolization in a retrospective analysis on 71 patients with unresectable HCC. Similarly, Carr et al found no significant difference in survival when comparing radioembolization and chemoembolization in patients with unresectable HCC. However, radioembolization was shown to be better than chemoembolization for down staging the disease, $58 \%$ for radioembolization vs $31 \%$ for chemoembolization (Lewandowski et al., 2009).

No significant survival difference has been shown between chemoembolization with drug-eluting beads loaded with doxorubicin and conventional chemoembolization. However, a higher rate of complete response, and disease control has been shown with chemoembolization with drug-eluting beads compared with conventional chemoembolization. Also, fewer liver and systemic adverse effects have been reported after chemoembolization with drug-eluting beads than with conventional chemoembolization.

\section{Future Directions}

With development of new drugs, improvement of intraarterial drug delivery techniques and knowledge of liver cancer biology, newer concepts in targeting liver cancer, and advances in imaging, the future of trans catheter therapies appears promising. These therapies in combination with other treatment modalities such as ablation or systemic therapies have great potential. In a study by Maluccio et al, there was no significant difference in the overall survival rates when compared surgery versus transarterial embolization in combination with ablation. Also, transarterial embolization in combination with ablation is effective in treating solitary HCC tumors up to $7 \mathrm{~cm}$ in selected patients (Wang et al., 2013). A new technology named nanotechnology, where drugs can be attached to tumor specific cells promises to be highly efficient method of drug delivery and gene therapies. New classes of drugs such as 3-bromopyruvate specifically targeting tumor metabolism delivered intraarterially could be more potent than conventional chemotherapeutic agents, and this new approach is extremely promising.
Recently, advances in the imaging such as catheterdirected CT angiography, C-arm angiographic CT, and combining DSA with MR imaging have successfully assisted superselective radioembolization, facilitating tumor targeting and sparing normal hepatic parenchyma. Traditionally, follow-up imaging is typically performed 4-6 weeks after therapy with radiologic measurement of tumor size as proposed by the WHO or RECIST guidelines. However, it has been recently stressed that the area of viable tumor, rather than the overall diameter is more important as transcatheter based therapies may lead to both anatomic and functional intratumoral changes. So it is essential that future-imaging evaluation of tumor response evaluate both anatomic and functional information of the tumor burden.

\section{Conclusions}

In conclusion, trans arterial therapies are the most appropriate means of treating the inoperable HCC. However, such treatments are only effective when appropriate selections on the patients are conducted. Although there may be complication arising from preferring these treatment options, there have never been any severe adverse effects on the patients. Thus, this makes the treatments safer if carried out professionally in accordance with the prescriptions. The unique aspects of all such therapies are their minimal toxicity profiles and highly effective tumor responses while normal hepatic parenchyma is spared. These unique characteristics provide an attractive therapeutic option for patients who may have previously had few alternatives. Despite major advances in techniques, imaging, and administration, further investigation needs to be performed.

\section{References}

Atassi B, Bangash AK, Bahram A, et al (2008). Multimodality imaging following Y-90 radioembolization: a comprehensive review and pictorial essay. Radio Graphics, 28, 81-99.

Brown DB, Fundakowski CE, Lisker-Melman M, et al (2004). Comparison of MELD and Child-Pugh scores to predict survival after chemoembolization for hepatocellular carcinoma. J Vasc Interv Radiol, 15, 1209-18.

Bruix J, Sherman M (2011) Management of hepatocellular carcinoma: An Update. Hepatol, 53, 1020-2.

Can A, Dogan E, Bayoglu IV, et al (2014). Multicenter epidemiologic study on hepatocellular carcinoma in Turkey. Asian Pac J Cancer Prev, 15, 2923-7.

Chang JM, Tzeng WS, Pan HB, Yang CF, Lai KH (1994). Transcatheter arterial embolization with or without cisplatin treatment of hepatocellular carcinoma: a randomized controlled-study. Cancer, 74, 2449-2453.

Coldwell DM, Stokes KR, Yakes WF (1994).. Embolotherapy: agents, clinical applications, and techniques. Radio Graphics, 14, 623-43.

Covey AM, Brody LA, Maluccio MA, Getrajdman GI, Brown KT (2002). Variant hepatic arterial anatomy revisited: digital subtraction angiography performed in 600 patients. Radiol, 224, 542-7.

Deodhar A, Covey AM, Thornton R, et al (2010). Safety and efficacy of transcatheter arterial embolization with particles only in the treatment of hepatocellular carcinoma with portal 
vein thrombus. J Intervention, 3, 3-11

Georgiades CS, Hong K, D’Angelo M, Geschwind JF (2005). Safety and efficacy of transarterial chemoembolization in patients with unresectable hepatocellular carcinoma and portal vein thrombosis. $J$ Vasc Interv Radiol, 16, 1653-9.

Hong K, Khwaja A, Liapi E, et al (2006). New intraarterial drug delivery system for the treatment of liver cancer: preclinical assessment in a rabbit model of liver cancer. Clin Cancer Res, 12, 2563-7.

Iñarrairaegui M, Thurston KG, Bilbao JI, et al (2010). Radioembolization with use of yttrium-90 resin microspheres in patients with hepatocellular carcinoma and portal vein thrombosis. J Vasc Interv Radiol, 21, 1205-12.

Jemal A, Bray F, Center MM, et al (2011). Global cancer statistics. CA Cancer J Clin, 61, 69-90.

Kan Z, McCuskey PA, Wright KC, Wallace S (1994). Role of Kupffer cells in iodized oil embolization. Invest Radiol, 29, 990-3

Kennedy A, Nag S, Salem R, et al (2007). Recommendations for radioembolization of hepatic malignancies using yttrium- 90 microsphere brachytherapy: a consensus panel report from the radioembolization brachytherapy oncology consortium. Int J Radiat Oncol Biol Phys, 68, 13-23

Khan G1, Hashim MJ (2015). Burden of virus-associated liver cancer in the Arab world, 1990-2010. Asian Pac J Cancer Prev, 16, 265-70.

Kim W, Clark TWI, Baum RA, Soulen MC (2001). Risk factors for liver abscess formation after hepatic chemoembolization. $J$ Vasc Interv Radiol, 12, 965-8

Kloeckner R, Otto G, Biesterfeld S, et al (2010). MDCT versus MRI assessment of tumor response after transarterial chemoembolization for the treatment of hepatocellular carcinoma. Cardiovasc Intervent Radiol, 33, 532-40

Kong WD, Cao JM, Xu J, et al (2012). Impact of low versus conventional doses of chemotherapy during transcatheter arterial chemoembolization on serum fibrosis indicators and survival of liver cancer patients. Asian Pac J Cancer Prev, 13, 4757-61.

Kruskal JB, Hlatky L, Hahnfeldt P, et al (1993). In vivo and in vitro analysis of the effectiveness of doxorubicin combined with temporary arterial occlusion in liver tumors. $J$ Vasc Interv Radiol, 4, 741-7.

Kulik LM, Carr BI, Mulcahy MF, et al (2008). Safety and efficacy of $90 \mathrm{Y}$ radiotherapy for hepatocellular carcinoma with and without portal vein thrombosis. Hepatol, 47, 71-81.

Lammer J, Malagari K, Vogl T, et al (2010). Prospective randomized study of doxorubicin eluting bead embolization in the treatment of hepatocellular carcinoma: results of the PRECISION V study. Cardiovasc Intervent Radiol, 33, 41-52

Lewandowski RJ, Kulik LM, Riaz A, et al (2009). A comparative analysis of transarterial downstaging for hepatocellular carcinoma: chemoembolization versus radioembolization. Am J Transplant, 9, 1920-8.

Liapi E, Geschwind JF (2011). Transcatheter arterial chemoembolization for liver cancer: is it time to distinguish conventional from drug eluting chemoembolization? Cardiovasc Intervent Radiol, 34, 37-49.

Llovet JM, Brú C, Bruix J (1999). Prognosis of hepatocellular carcinoma: the BCLC staging classification. Semin Liver Dis, 19, 329-38

Llovet JM, Real MI, Montaña X, et al (2002). Arterial embolisation or chemoembolisation versus symptomatic treatment in patients with unresectable hepatocellular carcinoma: a randomised controlled trial. Lancet, 359, 1734-9

Llovet JM, Ricci S, Mazzaferro V, et al (2008). Sorafenib in advanced hepatocellular carcinoma. N Engl J Med, 359, 378-90

Lo CM, Ngan H, Tso WK, et al (2002). Randomized controlled trial of transarterial Lipiodol chemoembolization for unresectable hepatocellular carcinoma. Hepatol, 35, 1164-71

Luo J, Guo RP, Lai E, et al (2011). Transarterial chemoembolization for unresectable hepatocellular carcinoma with portal vein tumor thrombosis: a prospective comparative study. Ann Surg Oncol, 18, 413-20.

Ma TC, Shao HB, Xu Y, Xu K (2013). Three treatment methods via the hepatic artery for hepatocellular carcinoma - a retrospective study. Asian Pac J Cancer Prev, 14, 2491-4.

Marelli L, Stigliano R, Triantos C, et al (2007). Transarterial therapy for hepatocellular carcinoma: which technique is more effective? A systematic review of cohort and randomized studies. Cardiovasc Intervent Radiol, 30, 6-25

Shi-Ying Wang, Wen-Hao Zhu, Sonya Vargulick, Sam Bill Lin, Zhi-Qiang Meng (2013). Nausea and vomiting after transcatheter arterial chemoembolization for hepatocellular carcinoma: incidence and risk factor analysis. Asian Pac J Cancer Prev, 14, 5995-6000

Song SY, Chung JW, Lim HG, Park JH (2006). Nonhepatic arteries originating from the hepatic arteries: angiographic analysis in 250 patients. J Vasc Interv Radiol, 17, 461-9.

Wang ZJ, Wang MQ, Duan F, et al (2013). Clinical application of transcatheter arterial chemoembolization combined with synchronous $\mathrm{C}$-arm cone beam $\mathrm{CT}$ guided radiofrequency ablation in treatment of large hepatocellular carcinoma. Asian Pac J Cancer Prev, 14, 1649-54.

Xie ZR1, Luo YL, Xiao FM, Liu Q, Ma Y (2015). Health related quality of life of patients with intermediate hepatocellular carcinoma after liver resection or transcatheter arterial chemoembolization. Asian Pac J Cancer Prev, 16, 4451-6.

Xu C, Lv PH, Huang XE, et al (2014). Safety and efficacy of sequential transcatheter arterial chemoembolization and portal vein embolization prior to major hepatectomy for patients with HCC. Asian Pac J Cancer Prev, 15, 703-6.

Yang J, Li C, Wen TF, et al (2014). Is hepatectomy for huge hepatocellular carcinoma $(\geq 10 \mathrm{~cm}$ in diameter) safe and effective? A single-center experience. Asian Pac J Cancer Prev, 15, 7069-77.

Zhang ZM, Zhang YM, Gao S, et al (2014). Treatment efficacy and prognostic factors for huge HCC based on Barcelona clinic liver cancer staging. Asian Pac J Cancer Prev, 15, 8823-8. 